\title{
Distribution of biglycan and decorin in rat dental tissue
}

D.M.H. Tenório ${ }^{1,2}$

M.F. Santos ${ }^{1}$ and T.M.T. Zorn ${ }^{1}$
1'Departamento de Histologia e Embriologia, Instituto de Ciências Biomédicas I, Universidade de São Paulo, São Paulo, SP, Brasil

${ }^{2}$ Departamento de Morfologia, Centro de Ciências Biológicas,

Universidade Federal de Alagoas, Maceió, AL, Brasil

\author{
Correspondence \\ T.M.T. Zorn \\ Laboratório de Biologia da \\ Reprodução \\ Departamento de Histologia e \\ Embriologia, ICB, USP \\ Av. Prof. Lineu Prestes, 1524 \\ 05508-900 São Paulo, SP \\ Brasil \\ Fax: +55-11-818-7309/818-7402 \\ E-mail: temtzorn@usp.br \\ Presented at SIMEC 2002 \\ (International Symposium \\ on Extracellular Matrix), \\ Angra dos Reis, RJ, Brazil, \\ October 7-10, 2002.
}

Research supported by CAPES and FAPESP. Part of a thesis presented by D.M.H. Tenório to the Institute of Biomedical Sciences, USP, São

Paulo, SP, Brazil, in partial fulfillment of the requirements

for the Doctoral degree.

$\ldots \ldots \ldots \ldots \ldots \ldots \ldots$

Received December 2, 2002

Accepted March 6, 2003

$\ldots \ldots \ldots \ldots \ldots \ldots$

\begin{abstract}
Biglycan and decorin are small leucine-rich proteoglycans that play several biological and structural roles in different tissues and organs. Several reports have indicated that biglycan participates in odontoblast and ameloblast differentiation and in the calcification process. In the present study we show that the expression of biglycan changes from within the ameloblasts and odontoblasts to the extracellular space according to the stage of animal development. In predentin and in the pulp space, however, biglycan was continually expressed throughout the period of investigation. In contrast, decorin was absent in odontoblasts and in ameloblasts and was exclusively expressed in predentin throughout the period of observation. In young rats, however, decorin was expressed in the extracellular spaces of the pulp, where it was concentrated mainly in the peripheral pulp.
\end{abstract}

Key words

- Decorin

- Biglycan

- Odontoblasts

- Predentin

- Immunohistochemistry
Proteoglycans consist of a central core protein to which variable numbers of glycosaminoglycan chains are covalently bound. The density, size and charge of proteoglycans are related to the maintenance and regulation of tissue functions (1). Proteoglycans mediate many functions during the developmental process, such as cellular proliferation, migration, adhesion and aggregation (2). The ability of proteoglycans to link to collagen is important for the formation and organization of collagen fibrils. Proteoglycans are also important for the control of inflammation, wound healing, calcification, and ion exchanges $(3,4)$. A number of studies have indicated that proteoglycans also influence mineralized tissues. They may have a regulatory role in the earliest stage of mineralization, during which mineral crystals are preferentially deposited in the gap zone of collagen fibrils and then grow into adjacent regions $(5,6)$. The mechanism of such activity is probably by changing the assembly, structure, and charge of the fibrils. Moreover, the sites of collagen that bind to calcium could be covered by proteoglycans and thus inhibit mineralization (7). Sulfated glycosaminoglycans possess the ability to bind cations like calcium due to their high anionic charge, and so enhance or inhibit the precipitation of calcium phosphate, depending on the calcium concentration $(4,8)$. Sulfated glycosaminoglycans also influence cytodifferentiation and play a role in stabilizing the differentiated state $(9,10)$.

Proteoglycans are present in the extracellular matrix of connective tissues such as the dental pulp. Due to their highly hydrophilic 
and polyanionic properties, one of the main functions of proteoglycans in the dental pulp is to regulate water retention and to protect it against high pressure.

Biglycan and decorin are small leucinerich proteoglycans that play several biological and structural roles. Their distribution and biological function vary significantly among tissues. Biglycan facilitates the initiation of apatite formation and inhibits its growth in a gelatin gel system (11). Previous results showed that this molecule has a role during the process of differentiation of odontoblasts and ameloblasts (12). Decorin is synthesized by odontoblasts and becomes associated with the pericellular environment. Decorin binds to collagen through its core protein (13), a structural property that may contribute to the initial assembly and aggregation of collagen as well as several other extracellular matrix components (14). A previous study (7) suggested that decorin also is a negative regulator of mineralization. The proposal of the present study was to analyze by immunocytochemistry the distribution of

Figure 1. Immunocytochemical detection of biglycan in dental germs of rats aged 5-11 days. On day 5 , the immunoreaction is usually weak (a). On day 7 a strong reaction is observed in odontoblasts and predentin and in the peripheral zone of the pulp (b). On day 9, a strong signal for biglycan is observed in ameloblasts, predentin and odontoblasts, whereas a weak signal is observed in the pulp (c). Higher magnification showing the reactivity in ameloblast cells at day 9 (d). On day 11 a slight reactivity for biglycan is observed in ameloblasts (e). Another view of day 11 showing that a strong reactivity remains in predentin, whereas the reaction in odontoblasts is weak (f). AMBs - ameloblasts; D - dentin; DG - dental germ; ODBs - odontoblasts; P pulp; PD - predentin.

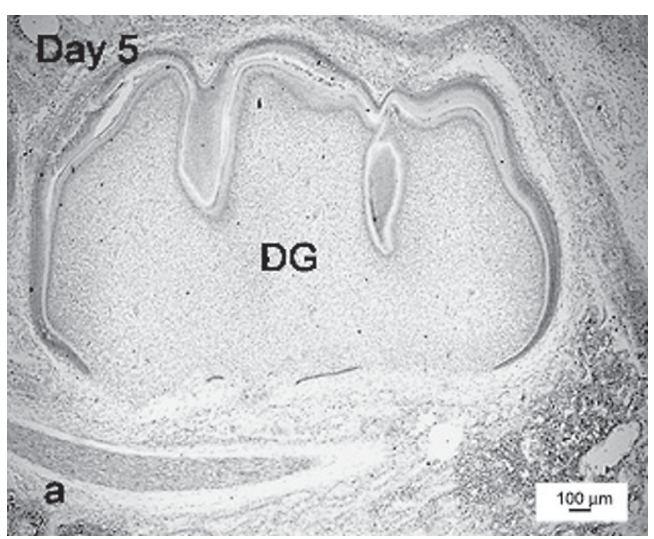

biglycan and decorin in different dental tissues during tooth development.

Wistar rats aged 5, 7, 9, and 11 days and 2 months were used. The animals were housed on a 12 -h light/dark schedule at $22^{\circ} \mathrm{C}$, with food and water available at all times. After sacrifice by decapitation, mandibles and maxillas were removed, dissected, and fixed in Bouin fixative solution for 1 day. Samples were decalcified in $4.13 \%$ EDTA for 3 weeks for the newborns and for 1 month for young rats, and embedded in Paraplast. Five-micrometer thick sections were cut, adhered to glass slides using $0.1 \%$ poly-L-lysine (Sigma, St. Louis, MO, USA), and then dried at room temperature.

During the immunocytochemistry procedure, each step was followed by a thorough rinse with phosphate-buffered saline (PBS). The sections were treated with $3 \% \mathrm{H}_{2} \mathrm{O}_{2}$ in PBS for 30 min to block endogenous peroxidase activity and then incubated for $1 \mathrm{~h}$ at $37^{\circ} \mathrm{C}$ in $20 \mathrm{mM}$ Tris- $\mathrm{HCl}$ buffer, $\mathrm{pH} 6.0$, containing $0.2 \mathrm{U} / \mathrm{ml}$ of chondroitinase AC II from Arthrobacter aurescens (Seikagaku
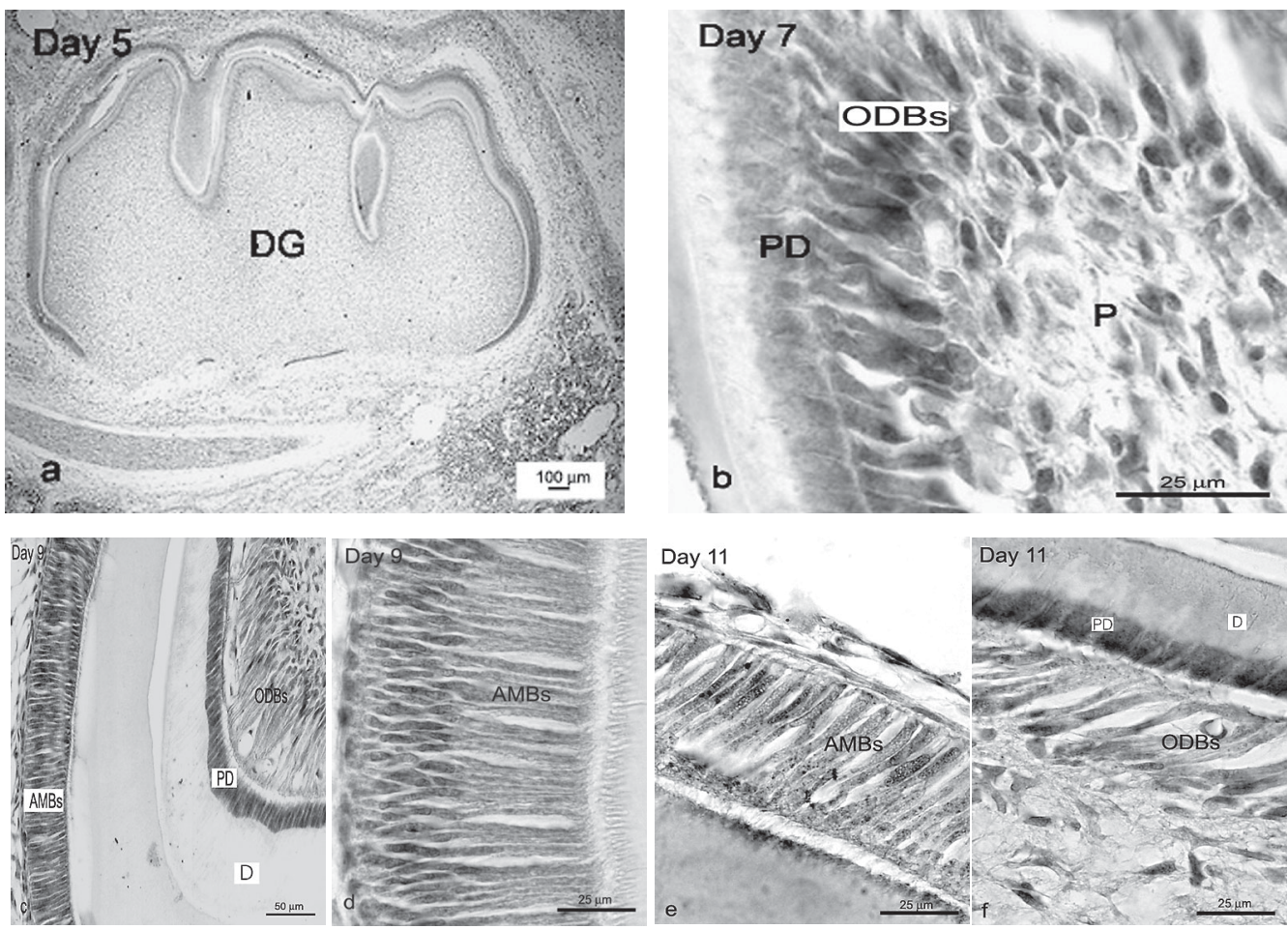
Corp., Tokyo, Japan). Nonspecific staining was blocked by incubating the section for 30 min with normal goat serum diluted $1: 1$ in PBS-10\% bovine serum albumin. Sections were incubated with the primary antibody, rabbit anti-decorin IgG, clone LF-113, diluted 1:2000 in PBS-0.3\% Tween 20 or rabbit anti-biglycan IgG, clone LF-106, diluted 1:1000 in PBS-0.3\% Tween 20 overnight at $4^{\circ} \mathrm{C}$, and controls omitting the primary antibody were included. Both antibodies were a generous gift from Dr. L.W. Fisher (National Institute of Dental and Craniofacial Research "NIDOR", NIH, Bethesda, MD, USA). The sections were incubated with goat anti-rabbit IgG conjugated with biotin (Vector Lab, Burlingame, CA, USA) diluted 1:1000 in PBS for $1 \mathrm{~h}$ at room temperature. After rinsing three times in PBS all sections were incubated with the streptavidin/peroxidase complex (Vector) for $1 \mathrm{~h}$ at room temperature. The reaction was visualized using $0.03 \%$ 3,3'-diaminobenzidine in PBS with $0.03 \%$ $\mathrm{H}_{2} \mathrm{O}_{2}$. After immunostaining, the sections were lightly stained with Mayer's hematoxylin. The sections were examined with a Nikon Eclipse E800 microscope and images were captured with an Image Pro Plus software (Media Cybernetics, Silver Spring, MD, USA) and subsequently printed with an Epson Stylus 777 printer.

As illustrated in Figure 1, at 5 days of age biglycan was observed in ameloblasts, odontoblasts, predentin and pulp. At 7 days of age a strong reaction for biglycan was present in ameloblasts, odontoblasts, and predentin. In the pulp, biglycan was observed in both extracellular spaces and cells. At 9 days of age ameloblasts and odontoblasts were less reactive, while intense staining for biglycan was observed in predentin. Biglycan was weakly expressed in the pulp extracellular spaces whereas it was not expressed in pulp cells. In 11-day-old rats biglycan was expressed intensely in predentin, while ameloblasts, odontoblasts and pulp cells were only slightly immunoreactive. In 2-month-old rats (data not shown), biglycan was limited to the predentin and pulp; no reaction for biglycan was detected in odontoblasts.

Figure 2 shows that, contrary to biglycan, decorin was absent in odontoblasts and was exclusively expressed in predentin throughout the study period. The immunoreaction
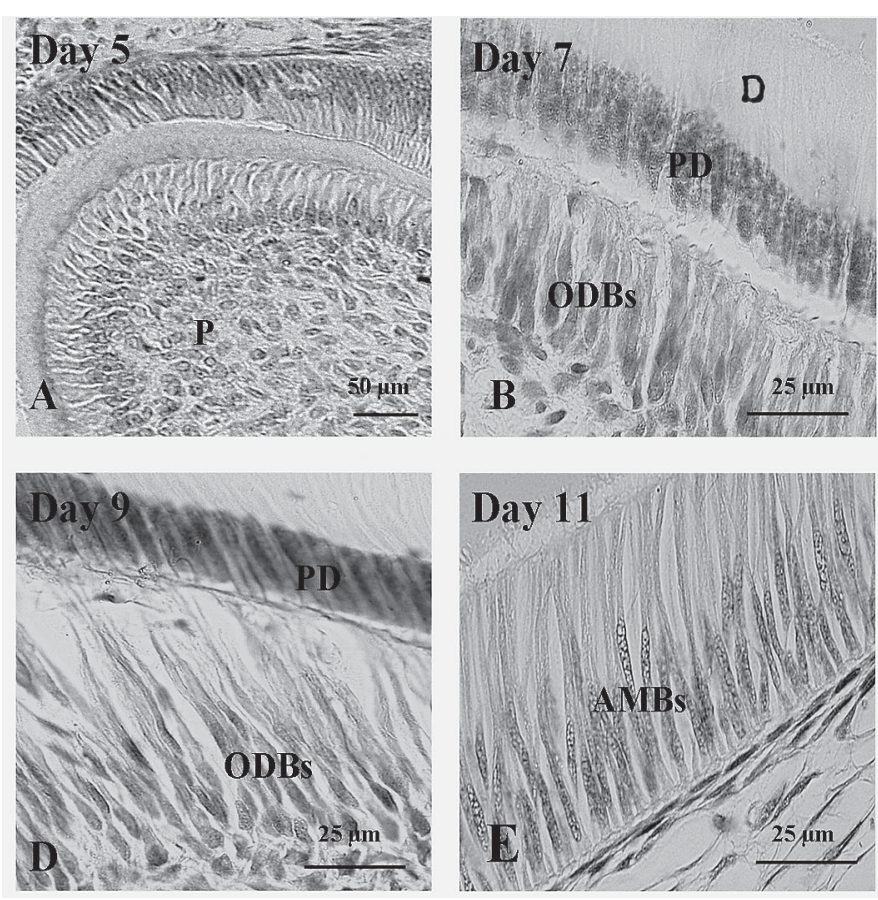
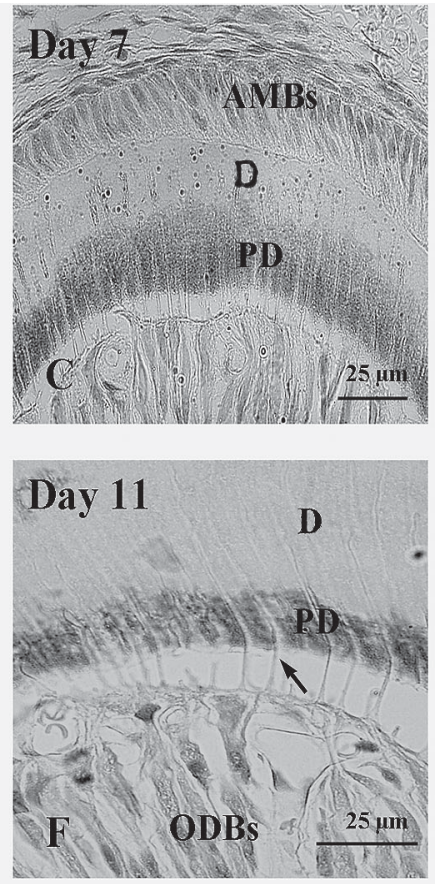

Figure 2. Immunocytochemical detection of decorin in dental germs of rats aged 5-11 days. On day 5 , the immunoreaction is very weak in predentin (A). On day 7 , a strong reactivity is observed in predentin (B). Another view showing the absence of immunoreactivity observed in ameloblasts (C). On day 9, the immunoreaction is not observed in odontoblasts, whereas it is strong in predentin (D). On day 11, ameloblasts continue to be negative (E). Another view of the 11 th day showing a decrease in the reactivity in predentin (F). AMBs - ameloblasts; arrow odontoblastic process; D - dentin; ODBs - odontoblasts; P pulp; PD - predentin. 
for decorin was weak on day 5 of age and progressively increased on day 7 and day 9 . On day 11 the reaction decreased in predentin. In young 2-month-old rats decorin was expressed in the extracellular spaces of the pulp, where it was concentrated mainly in the peripheral pulp and was continually expressed in predentin (data not shown).

In the present study we observed biglycan in the ameloblasts and odontoblasts during the early stages of tooth development. During tooth development, groups of cells are induced to differentiate by interactions with neighboring cells and tissues. Moreover, the inductive interactions between tissues must involve extracellular matrix molecules and growth factors. Several studies have suggested a role for biglycan during the process of differentiation, probably by interaction with TGF- $\beta$, as this molecule promotes an increase in the amount of biglycan in vitro (15). Biglycan is also important in cell attachment and proliferation (16), events that occur during tooth development. The sustained expression of biglycan in predentin suggests a structural role of this molecule by interaction with collagen fibrils (17). As biglycan was detected in ameloblasts, we may consider that this molecule has a role in the process of amelogenesis, including ameloblast differentiation and enamel formation (12).
In the present study we detected a transient expression of decorin. Initially, decorin was observed solely in predentin, and later in dental pulp. Decorin may be involved in dentin formation via its specific association with type I collagen fibrils, implying a role in fibril growth and assembly (13). The expression of decorin in the pulp of young rats may contribute to the undifferentiated state of some cells and to the maintenance of the unmineralized state of pulp tissue. A possible role for decorin as an inhibitor of mineralization has been reported (18). Decorin may occupy the mineralization sites blocking the interaction between collagen and the molecules that initiate mineralization, thus regulating the pattern of collagen mineralization, like other collagenbinding small leucine-rich proteoglycans (19). The exact role of biglycan and decorin in dental tissues, however, is still controversial.

\section{Acknowledgments}

We gratefully acknowledge Dr. Alison Colqhoun for critically reading the manuscript. We are grateful to Dr. Larry Fisher (National Institutes of Health, NIH, Bethesda, MD, USA) for donating the antibodies and to Cleusa Maria Raspantini Pellegrini for technical support.

\section{References}

1. Bartold PM \& Schlagenhauf U (1995). Localization of chondroitin sulphate and dermatan sulphate in human dental pulps - an immunohistochemical study. International Endodontic Journal, 28: 19-25.

2. Galbraith DB, Cutler LS \& Kollar EJ (1992). The correlation of temporal regulation of glycosaminoglycan synthesis with morphogenetic events in mouse tooth development. Archives of Oral Biology, 37: 623-628.

3. Diegelmann RF, Cohen I \& McCoy B (1979). Growth kinetics and collagen synthesis of normal skin, normal scar and keloid fibroblasts in vitro. Journal of Cellular Physiology, 98: 341-346.

4. Linde A (1973). A study of the dental pulp glycosaminoglycans from permanent human teeth and rat and rabbit incisors. Archives of Oral Biology, 18: 49-59.

5. Cheng H, Caterson B, Neame PJ, Lester GE \& Yamauchi M (1996).
Differential distribution of lumican and fibromodulin in tooth cementum. Connective Tissue Research, 34: 87-96.

6. Cheng H, Caterson B, Neame PJ, Lester GE \& Yamauchi M (1999). Identification and immunolocalization of chondroitin sulfate proteoglycans in tooth cementum. Connective Tissue Research, 40: 37-47.

7. Hoshi K, Kemmotsu S, Takeuchi Y, Amizuka N \& Ozawa H (1999). The primary calcification in bones follows removal of decorin and fusion of collagen fibrils. Journal of Bone and Mineral Research, 14: 273-280.

8. Schubert M \& Pras M (1968). Ground substance protein-polysaccharides and the precipitation of calcium phosphate. Clinical Orthopaedics, 60: 235-255

9. Cutler LS (1990). The role of extracellular matrix in the morphogenesis and differentiation of salivary glands. Advances in Dental Re- 
search, 4: 27-33.

10. Cutler LS \& Christian CP (1984). Inhibition of rat salivary gland adenylate cyclase by glycosaminoglycans and high molecular weight polyanions. Archives of Oral Biology, 29: 629-633.

11. Boskey AL, Spevak L, Doty SB \& Rosenberg L (1997). Effects of bone CS-proteoglycans, DS-decorin, and DS-biglycan on hydroxyapatite formation in a gelatin gel. Calcified Tissue International, 61: 298-305.

12. Matsuura T, Duarte RW, Cheng Hui, Uzawa K \& Yamauchi M (2001). Differential expression of decorin and biglycan genes during mouse tooth development. Matrix Biology, 12: 197-201.

13. Vogel KG, Paulsson M \& Heinegard D (1984). Specific inhibition of type I and type II collagen fibrogenesis by small proteoglycans of tendon. Biochemical Journal, 223: 587-597.

14. Öbrink B \& Sundelof LO (1973). Light scattering in the study of associating macromolecules. The binding of glycosaminoglycans to collagen. European Journal of Biochemistry, 37: 226-232.

15. Kähäri VM, Larjava H \& Uitto J (1991). Differential regulation of extracellular matrix proteoglycan gene expression. Journal of Biological Chemistry, 266: 608-615.

16. Bianco P, Fisher LW, Young MF, Termine JD \& Gehron Robey P (1990). Expression and localization of the two small proteoglycans biglycan and decorin in developing human skeletal and non-skeletal tissue. Journal of Histochemistry and Cytochemistry, 38: 1549-1563.

17. Schönherr E, Witsch-Prehm P, Harrach B, Robenek H, Rauterberg J \& Kresse $H$ (1995). Interaction of biglycan with type I collagen. Journal of Biological Chemistry, 270: 2776-2783.

18. Yamauchi M, Uzawa K, Katz EP, Lopes MM, Verdelis K \& Cheng H (2000). Distribution of small keratan sulfate proteoglycans in predentin and dentin. In: Goldberg M, Boskey A \& Robinson C (Editors), Chemistry and Biology of Mineralized Tissues. American Academy of Orthopedic Surgeons, Rosemont, IL, USA, 305-310.

19. Embery G, Hall R, Waddington R, Septier D \& Goldberg M (2001). Proteoglycans in dentinogenesis. Critical Reviews in Oral Biology and Medicine, 12: 331-349. 